Check for updates

Cite this: Chem. Sci., 2019, 10, 5807

๑ All publication charges for this article have been paid for by the Royal Society of Chemistry

\title{
Continuous nonenzymatic cross-replication of DNA strands with in situ activated DNA oligonucleotides $\uparrow$
}

\author{
Evgeniia Edeleva, (D) ${ }^{a}$ Annalena Salditt, (iD ${ }^{a}$ Julian Stamp, (D) ${ }^{\text {a }}$ Philipp Schwintek, (D) ${ }^{a}$ \\ Job Boekhoven (D) ${ }^{b}$ and Dieter Braun (D) *a
}

\begin{abstract}
Continuous enzyme-free replication of oligonucleotides is central for open-ended evolution experiments that mimic the origin of life. Here, we studied a reaction system, whereby two 24 mer DNA templates crosscatalyzed each other's synthesis from four 12mer DNA fragments, two of which were in situ activated with the condensing agent 1-ethyl-3-(3-dimethylamino-propyl)carbodiimide (EDC). We circumvented the problem of product inhibition by melting the stable product duplexes for their reuse as templates in the following ligation step. The system reproduced itself through ligation/melting cycles and survived exponential dilution. We quantified EDC-induced side reactions in a detailed kinetic model. The model allowed us to analyze the effects of various reaction rates on the system's kinetics and confirmed maximal replication under the chosen conditions. The presented system enables us to study nonenzymatic open-ended evolution experiments starting from diverse sequence pools.
\end{abstract}

Received 14th February 2019

Accepted 28th April 2019

DOI: $10.1039 /$ c9sc00770a

rsc.li/chemical-science isothermal conditions. ${ }^{14,15}$ This isothermal replication was limited to short strands.

Templated ligation of longer strands at a constant temperature would lead to the formation of stable product duplexes inhibiting further strand replication. ${ }^{16}$ This stable product formation is known as a problem of product inhibition. Additionally, the studies of von Kiedrowski used a more efficient replication with a phosphoramidate bond forming at the ligation site instead of the naturally occurring phosphodiester bond. ${ }^{17}$ DNA systems with a phosphoramidate bond are not biologically motivated and would be incompatible with the deep sequencing analysis required to study sequence space evolution from random strands.

Here, we studied replication-by-ligation of $12 \mathrm{mer}$ oligonucleotide fragments on 24 mer templates using EDC. EDC is used in the origin of life field as a model molecule to study polymerization, replication, and ligation under abiotic conditions. ${ }^{18-21}$ The chosen ligation chemistry allowed us to form natural phosphodiester bonds at the ligation site. To overcome product inhibition, we used temperature cycling ${ }^{22}$ as a possible nonequilibrium setting for the origin of life. Such a setting could have existed on the early Earth in temperature gradients across water filled porous volcanic rocks. ${ }^{23,24}$

The nonenzymatic chemistry under high temperature conditions makes side reactions a significant possibility. However, side reactions are likely inevitable in any complex environment due to the prebiotic clutter. ${ }^{25}$ We showed that our system reproduced over several ligation/melting cycles and survived the exponential dilution conditions despite the side reactions. Thus, temperature cycling as a means to overcome product inhibition in the absence of enzymes seems to be a realistic possibility.
${ }^{a}$ Systems Biophysics, Physics Department, Ludwig-Maximilians-Universität München, Amalienstraße 54, 80799 München, Germany. E-mail: dieter.braun@lmu.de

${ }^{b}$ Chemistry Department and Institute for Advanced Study, Technical University of Munich, Lichtenbergstraße 4, 80895 Garching, Germany

$\dagger$ Electronic supplementary information (ESI) available: Figures, details of data quantification, HPLC-MS analysis, and the kinetic model and simulation. See DOI: $10.1039 / \mathrm{c} 9 \mathrm{sc} 00770 \mathrm{a}$ 


\section{Results and discussion}

\section{Experimental system}

We implemented our system ("Input DNA" in Fig. 1a) with two 24mer DNA templates ab and BA and complementary fragments A (12mer), B (15mer, with 12 bases binding to ab), b (12mer), and $\sim \mathrm{a}$ (21mer, with 12 bases binding to BA). The template sequences consisted of adenine and thymine nucleobases and did not form hairpins. The strands $\sim$ a (tagged a) and $\sim \mathrm{B}$ (tagged B) contained polycytidine tags at the $5^{\prime}$-ends for individual detection via electrophoresis (Fig. S1b $\dagger$ ). Phosphate groups at the $3^{\prime}$-ends of $\sim \mathrm{a}$ and $\sim \mathrm{B}$ allowed for the ligation reaction.

Addition of EDC started a nonenzymatic ligation of the fragments on the corresponding templates ("Initial Ligation" in Fig. 1a), producing the tagged replicates $\sim \mathrm{BA}(27$ mer with a $3 \mathrm{nt}$ tag) and $\sim \mathrm{ab}$ (33mer with a 9nt tag) with a phosphodiester bond at the ligation site. Once the replicates formed, they were crosscatalytically replicated with the help of temperature cycling ("Exponential Replication" in Fig. 1a).

\section{Surviving replicator}

To monitor the reaction, we ran aliquots from different time points on a Bioanalyzer instrument (Fig. 1c) and quantified product formation by fitting Gaussian functions as described in ESI 1. $\dagger$ We observed that the concentration of each motif (BA + $\sim \mathrm{BA}$ or $\mathrm{ab}+\sim \mathrm{ab}$ ) consistently increased (Fig. 2a). Due to the hourly dilution scheme, the concentration of the initial templates at the same time decreased exponentially. In total, each motif amplified (2.2 \pm 0.5 )-fold within 6 hours (Table S1†), while only $59 \%$ of the original templates were still present after 6 hours. Therefore, despite diluting the templates, the information-containing motifs $\mathrm{ab}$ and BA survived in the system.

Without the $3^{\prime}$-phosphates (Fig. S2a $\dagger$ ) or in the absence of templates (Fig. S2b $\dagger$ ), the reaction did not produce clear product peaks. The survival of the replicating strands depended critically on temperature cycling. In its absence, the system could not overcome product inhibition and the replicating strands slowly diluted under the feeding/dilution conditions of the reaction (Fig. 2b).

To make sure that the reaction products acted as templates as reaction progressed, we also started the reaction with one template BA. In this case, $\sim$ BA could form only after $\sim \mathrm{ab}$ formed. Indeed, we observed the formation of $\sim \mathrm{ab}$ that then triggered the formation of $\sim$ BA (Fig. 1d).

These results indicate that 24nt-long DNA strands with defined sequences can replicate by ligation in a templated fashion under simple in situ activation conditions. Similar conditions could also support oligonucleotide polymerization $^{\mathbf{1 8 - 2 0}}$ as a possible source of short strands on the early Earth.

\section{EDC-driven side reactions}

When carbodiimides, such as EDC, are used for condensation of carboxylic acids with weak nucleophiles, like carboxylic acids or pyrophosphates, ${ }^{19}$ an unwanted side reaction arises as a consequence of the relatively low nucleophilicity. In the side (a)

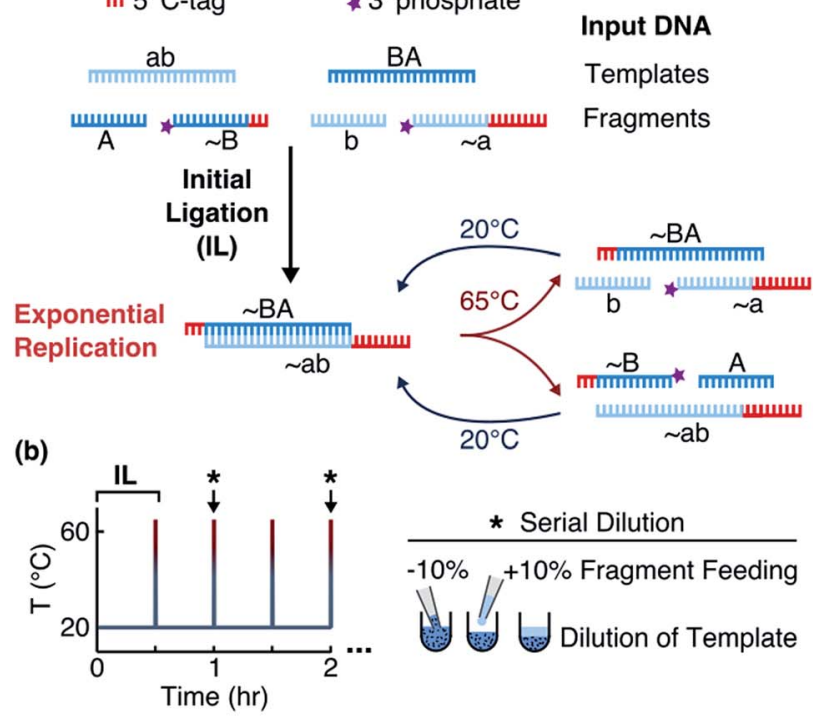

(c)
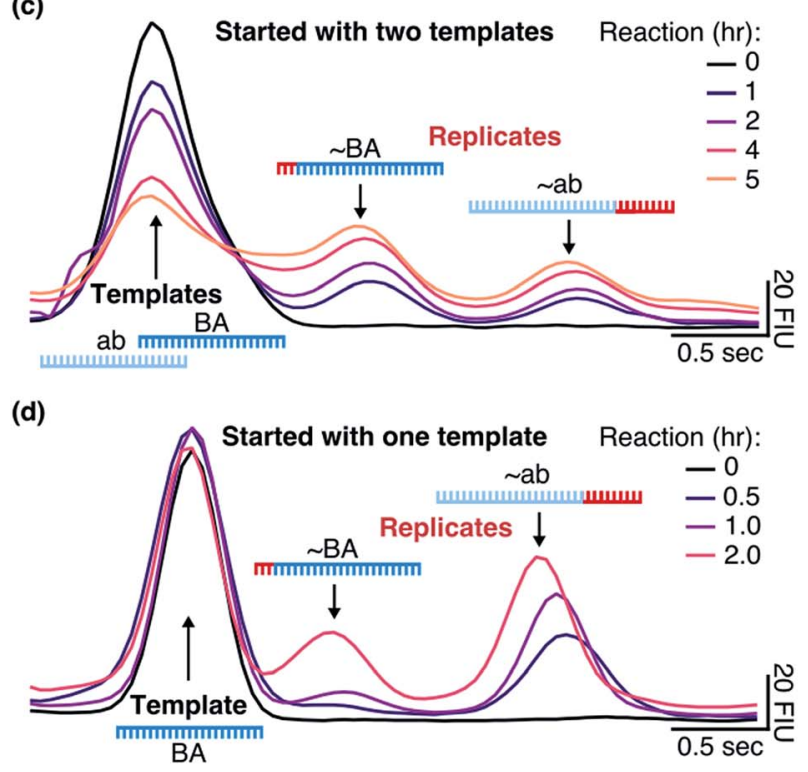

Fig. 1 Replication system and detection. (a) When the templates were mixed with the fragments and EDC at $20^{\circ} \mathrm{C}$, an initial ligation (IL) occurred that formed the replicates $\sim \mathrm{BA}$ and $\sim \mathrm{ab}$. By implementing temperature cycling, the replicates were then exponentially amplified. (b) The reaction was cycled between $20^{\circ} \mathrm{C}$ for 0.5 hours and $65^{\circ} \mathrm{C}$ for 1 second with the heating and cooling rates of 8 and $6{ }^{\circ} \mathrm{C} \mathrm{s}^{-1}$, respectively. Every hour (black star and arrow), 10\% of the reaction volume was replaced with $10 \%$ of the fragment mix in the reaction buffer. (c) Tagged replicates separated from the templates on a microfluidic gel electrophoresis chip. Reactions were started with 1 $\mu \mathrm{M}$ of each template and $10 \mu \mathrm{M}$ of each fragment in the reaction buffer and the aliquots were taken after $0,1,2,4$, and 5 hours. (d) The same as in (c) but reactions were started with $1 \mu \mathrm{M}$ of only template BA and aliquots were taken after $0,0.5,1$, and 2 hours. Additional details are explained in ESI 1.†

reaction, the O-acylisourea intermediate rearranges into the stable non-reactive $\mathrm{N}$-acylurea. ${ }^{20}$

To search for signs of side reactions in our system, we took a closer look at the fragments incubated with EDC over time. We 

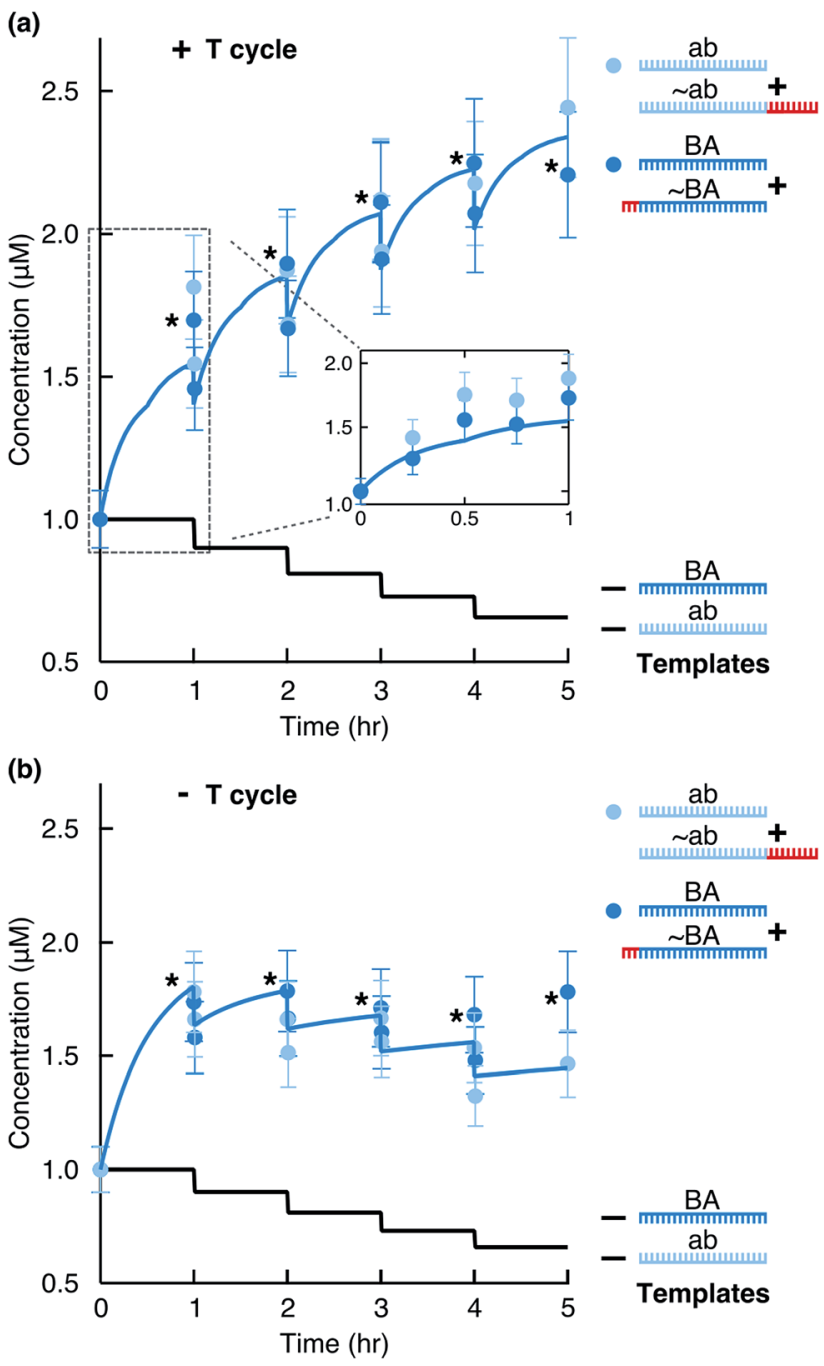

Fig. 2 Replication by ligation. The stars mark data points just before a dilution step. The solid blue line shows the full kinetics simulation of the system. The solid black line indicates the calculated concentration of each template considering the dilution steps. (a) Concentration of replicates from Fig. 1c quantified for the total ab and BA motifs (light and dark blue circles). For hours 1, 2, 3, and 4, concentrations before and after the dilution steps were quantified. The inset shows time points taken within the first hour and the simulation result for the first hour. (b) The reaction performed in the absence of temperature cycling but with dilution/feeding steps.

observed that the fragments bearing $3^{\prime}$-phosphate groups decreased in concentration and a shoulder formed next to the peak assigned to fragment $\sim$ a (Fig. 3a). Shoulder formation only occurred in the presence of the 3 -phosphate group (Fig. S3b†), suggesting that it originated from a side reaction between EDC and the $3^{\prime}$-phosphate group (Fig. S3c $\dagger$ ). Since the EDC-modified fragments are less negatively charged and 155 Da heavier than unmodified fragments, they were expected to run slower in a gel electrophoresis experiment, confirmed by the position of the shoulder on the right of the peak. Additionally, we identified in the HPLC-MS analysis the masses for an ' $\sim$ a + EDC' side product (corresponding to the structure in Fig. S3c $\dagger$ ) and for a circularization product of $\sim$ a (Fig. S4, ESI 2, Table S2 $\dagger$ ). (a)

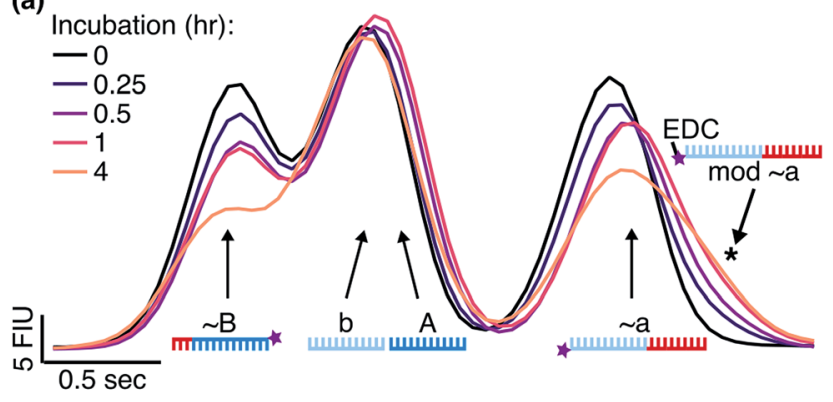

(b)

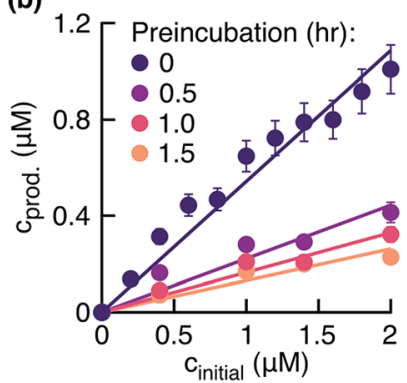

(c)

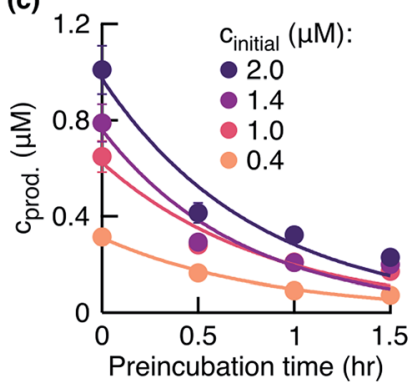

Fig. 3 Effect of fragment side reaction on the ligation rate. (a) Fragments were incubated in the reaction buffer under the temperature cycling conditions. Peaks were identified using the ladder peak (Fig. S3a †). The signal from the phosphorylated fragments decreased and a shoulder formed on the right of the $\sim a$ peak (black star). (b) Product concentrations 0.5 hours after the start of the reaction plotted against initial template concentrations. Reactions were started with fragments preincubated in the reaction buffer for $0,0.5,1$, and 1.5 hours before the addition of templates. Linear fits gave ligation rates $k_{\text {lig }}$ of $1.08 \pm 0.04,0.44 \pm 0.04,0.32 \pm 0.02$, and $0.26 \pm 0.02 \mathrm{~h}^{-1}$, respectively. The respective replication yields were $(61 \pm 10) \%$, (28 \pm $10) \%,(19 \pm 4) \%$, and (15 \pm 3$) \%$ in 0.5 hours. (c) Data from (b) replotted to demonstrate the exponential decrease in product concentrations during the preincubation in EDC. The exponential fits yielded the rate constants of $1.14 \pm 0.12,1.14 \pm 0.24,1.38 \pm 0.36$, and $1.26 \pm 0.24 \mathrm{~h}^{-1}$ for the initial template concentrations of $2.0,1.4,1.0$, and $0.4 \mu \mathrm{M}$, respectively.

The fragment $\sim \mathrm{B}$ decreased in concentration over time as well (Fig. 3a). This result suggested that EDC also modified $\sim \mathrm{B}$ at its $3^{\prime}$-phosphate group. The expected shoulder in this case was overlaid by the dominant peak combined from the individual peaks of fragments $\mathrm{b}$ and A. Our system successfully replicated despite the observed side reaction (Fig. 2a).

In the next step, we investigated and compared the influence of the ligation and side reaction rates in the system. To measure the ligation rate under the reaction conditions, we followed the reactions with varied initial template concentrations for 0.5 hours and quantified the product concentrations (Fig. 3b, dark purple filled circles). The product concentration showed linear dependence on the initial template concentration, suggesting underlying exponential behavior of the reaction. The ligation rate obtained from the linear fit was $1.08 \pm 0.04 \mathrm{~h}^{-1}$. The experiment resulted in a replication yield of $(61 \pm 10) \%$ in 0.5 hours, where the replication yield is defined as the replicate concentration at time $t$ divided by the template concentration at time $t=0$. 
To quantify the effect of the side reaction on the ligation rate, we next incubated the fragments in EDC for $0.5,1.0$, or 1.5 hours in the absence of templates under the temperature cycling conditions of the reaction. After the so-called preincubation time without templates, we added the template strands to start the ligation reaction. The ligation rate decreased to $0.44 \pm 0.04,0.32 \pm 0.02$, and $0.26 \pm 0.02 \mathrm{~h}^{-1}$ after 0.5, 1.0, and 1.5 hours of preincubation, respectively (Fig. 3b). Respective replication yields were $(28 \pm 10) \%,(19 \pm 4) \%$, and $(15 \pm$ $3) \%$ in 0.5 hours. For a preincubation time of 1.5 hours, the replication rate and yield decreased 4 -fold compared to no preincubation.

To approximate the side reaction rate, we studied the product concentration decrease over the fragment preincubation time (Fig. 3c). The exponential fits with assumed zero asymptote averaged to an effective first order rate constant of $1.23 \pm 0.11 \mathrm{~h}^{-1}$ with the error given as the standard deviation. Despite the same order of magnitude between the ligation and side reaction rates, the designed system survived the exponential dilution and successfully replicated for several ligation/ melting cycles.

\section{Kinetic model of the replication reaction}

The design and further optimization of replication systems requires a deeper understanding of the underlying kinetic behavior. We modeled the reaction with a set of rate equations (eqn (1)-(23)). Eqn (1)-(4) describe the reversible formation of the intermolecular complexes between templates and their complementary fragments with association and dissociation rate constants $k_{\text {on }}^{12 n t}$ and $k_{\text {off }}^{12 n t, T}$, where $T$ refers to the temperature dependence of the dissociation rate. Following the formation of the complex, an irreversible ligation reaction occurs with a ligation rate $k_{\text {lig }}$ (eqn (5) and (6)). We did not differentiate between the ligation kinetics for templates and replicates that contained tags since the tags had little effect on the ligation reaction (Fig. 4a). The resulting complex reversibly dissociates with dissociation and association rate constants $k_{\text {off }}^{24 n t, T}$ and $k_{\text {on }}^{24 n t}$ (eqn (7)). Complementary fragments also reversibly bind to each other with association and dissociation rate constants $k_{\text {on }}^{12 n t}$ and $k_{\text {off }}^{12 \text { nt, } T}$ (eqn (8) and (9)).

$$
\begin{gathered}
\mathrm{BA}+\mathrm{a}+\mathrm{b} \underset{k_{\mathrm{off}}^{12 \mathrm{nt}}}{\stackrel{k_{\mathrm{on}}^{12 \mathrm{nt}}}{\rightleftharpoons}}[\mathrm{BA} \cdot \mathrm{a}]+\mathrm{b} \underset{k_{\mathrm{off}}^{12 \mathrm{nt}}}{\stackrel{k_{\mathrm{on}}^{12 \mathrm{nt}}}{\rightleftharpoons}}[\mathrm{BA} \cdot \mathrm{a} \cdot \mathrm{b}] \\
\mathrm{BA}+\mathrm{a}+\mathrm{b} \underset{k_{\mathrm{off}}^{12 \mathrm{nt}}}{\stackrel{k_{\mathrm{on}}^{12 \mathrm{nt}}}{\rightleftharpoons}}[\mathrm{BA} \cdot \mathrm{b}]+\mathrm{a} \underset{k_{\mathrm{off}}^{12 \mathrm{nt}}}{\stackrel{k_{\mathrm{on}}^{12 \mathrm{nt}}}{\rightleftharpoons}}[\mathrm{BA} \cdot \mathrm{a} \cdot \mathrm{b}] \\
\mathrm{ab}+\mathrm{A}+\mathrm{B} \underset{k_{\mathrm{off}}^{12 \mathrm{nt}}}{\stackrel{k_{\mathrm{on}}^{12 \mathrm{nt}}}{\rightleftharpoons}}[\mathrm{A} \cdot \mathrm{ab}]+\mathrm{B} \underset{k_{\mathrm{off}}^{12 \mathrm{nt}}}{\stackrel{k_{\mathrm{on}}^{12 \mathrm{nt}}}{\rightleftharpoons}}[\mathrm{B} \cdot \mathrm{A} \cdot \mathrm{ab}] \\
\mathrm{ab}+\mathrm{A}+\mathrm{B} \underset{k_{\mathrm{off}}^{12 \mathrm{nt}}}{\stackrel{k_{\mathrm{on}}^{12 \mathrm{nt}}}{\rightleftharpoons}}[\mathrm{B} \cdot \mathrm{ab}]+\mathrm{A} \underset{k_{\mathrm{off}}^{12 n \mathrm{nt}}}{\stackrel{k_{\mathrm{on}}^{12 \mathrm{nt}}}{\rightleftharpoons}}[\mathrm{B} \cdot \mathrm{A} \cdot \mathrm{ab}] \\
{[\mathrm{BA} \cdot \mathrm{a} \cdot \mathrm{b}] \stackrel{k_{\mathrm{lig}}}{\rightarrow}[\mathrm{BA} \cdot \mathrm{ab}]}
\end{gathered}
$$

-

- $\mathrm{m! \textrm {II }}$ (a)

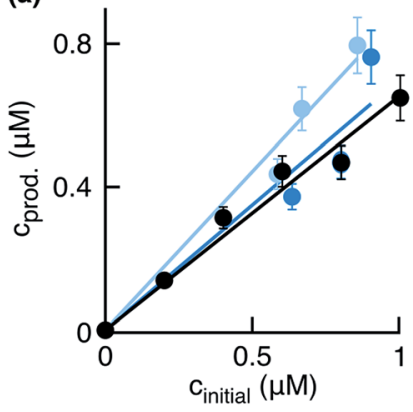

(b)
Fig. 4 Ligation rate for the simulation. (a) Product concentrations 0.5 hours after the start of the reaction. Templates were replaced with $\sim a b$ or $\sim \mathrm{BA}$ (light and dark blue points) for the initial ligation, or the initial ligation was started with templates as before (black points). The ligation rates from the linear fits were $1.78 \pm 0.08,1.40 \pm 0.14,1.32 \pm 0.06$ $\mathrm{h}^{-1}$, respectively. Respective replication yields were $(87 \pm 11) \%,(67 \pm$ $15) \%$, and $(55 \pm 6) \%$ in 0.5 hours. (b) Product concentrations quantified $10 \mathrm{~min}$ after the start of the reaction. The linear fit produced a ligation rate of $2.16 \pm 0.12 \mathrm{~h}^{-1}$, with a replication yield of $(39 \pm 7) \%$ in 10 minutes.

$$
\begin{gathered}
{[\mathrm{B} \cdot \mathrm{A} \cdot \mathrm{ab}] \stackrel{k_{\text {lig }}}{\longrightarrow}[\mathrm{BA} \cdot \mathrm{ab}]} \\
{[\mathrm{BA} \cdot \mathrm{ab}] \underset{k_{\mathrm{on}}^{24 \mathrm{nt}}}{\stackrel{k_{\mathrm{on}}^{24 \mathrm{nt}}}{\rightleftharpoons}} \mathrm{BA}+\mathrm{ab}} \\
\mathrm{A}+\mathrm{a} \underset{k_{\mathrm{off}}^{12 \mathrm{nt}}}{\stackrel{k_{\mathrm{on}}^{12 \mathrm{nt}}}{\rightleftharpoons}}[\mathrm{A} \cdot \mathrm{a}] \\
\mathrm{B}+\mathrm{b} \underset{k_{\mathrm{off}}^{12 \mathrm{nt}}}{\stackrel{k_{\mathrm{on}}^{12 \mathrm{nt}}}{\rightleftharpoons}}[\mathrm{B} \cdot \mathrm{b}]
\end{gathered}
$$

We observed in the previous experiments that the replication rate slowed down during the first 30 minutes of the reaction (Fig. 2a, inset). We assumed that the product inhibition and side reactions had already affected the reaction, and the ligation rate quantified from these data was being underestimated. To obtain the value of $k_{\text {lig }}$ independent of the product inhibition and side reaction effects, we measured product concentrations $10 \mathrm{~min}$ after the start of the reaction for different initial template concentrations (Fig. 4b). The linear fit gave a ligation rate of $2.16 \pm 0.12 \mathrm{~h}^{-1}$, about two-fold higher than when measuring product formation after 30 minutes.

We modeled the association rate as length-independent and obtained the value for $k_{\mathrm{on}}^{12 \mathrm{nt}}$ and $k_{\text {on }}^{24 \mathrm{nt}}$ from the literature ${ }^{26,27}$ (ESI $3 \dagger)$. The rates $k_{\text {off }}^{12 n t, 20^{\circ} \mathrm{C}}, k_{\text {off }}^{12 n t, 65^{\circ} \mathrm{C}}, k_{\text {off }}^{24 n t, 20^{\circ} \mathrm{C}}$, and $k_{\text {off }}^{24 n t, 65^{\circ} \mathrm{C}}$ were estimated from the analysis of melting curves, as described in detail in ESI 3. $\uparrow$ We did not explicitly model the hydrolysis of EDC because a more than 2500-fold excess of EDC over DNA is expected to be present at the end of the reaction ${ }^{28-30}$ (ESI $3 \dagger$ ). To model feeding and dilution, we introduced rates $k_{\text {feed }}$ and $k_{\text {dil }}$ defined by the experimental feeding and dilution protocol. 
We next added side reaction pathways to the system of equations. Based on the experimental observation (Fig. 3a, S4 $\dagger$ ), we allowed the fragments ' $\mathrm{B}$ ' and ' $\mathrm{a}$ ' to turn into side products with a side reaction rate $k_{\text {s.r. }}$ independent of the fragments being in a free or a bound state (eqn (10)-(17)). The resulting fragments $\mathrm{B}^{*}$ and $\mathrm{a}^{*}$ still bind to complementary fragments, templates, and replicates (eqn (18)-(23)).

$$
\begin{aligned}
& \mathrm{B} \stackrel{k_{\text {s.r. }}}{\longrightarrow} \mathrm{B} * \\
& \mathrm{a} \stackrel{k_{\text {s.r. }}}{\longrightarrow} \mathrm{a}^{*} \\
& {[\mathrm{~B} \cdot \mathrm{ab}] \stackrel{k_{\text {s.r. }}}{\longrightarrow}[\mathrm{B} * \cdot \mathrm{ab}]} \\
& {[\mathrm{BA} \cdot \mathrm{a}] \stackrel{k_{\text {s.r. }}}{\longrightarrow}[\mathrm{BA} \cdot \mathrm{a} *]} \\
& {[\mathrm{B} \cdot \mathrm{A} \cdot \mathrm{ab}] \stackrel{k_{\mathrm{s.r}}}{\longrightarrow}[\mathrm{B} * \cdot \mathrm{A} \cdot \mathrm{ab}]} \\
& {[\mathrm{BA} \cdot \mathrm{a} \cdot \mathrm{b}] \stackrel{k_{\text {s.r. }}}{\longrightarrow}[\mathrm{BA} \cdot \mathrm{a} * \mathrm{~b}]} \\
& {[\mathrm{B} \cdot \mathrm{b}] \stackrel{k_{\text {s.r. }}}{\longrightarrow}\left[\mathrm{B}^{*} \cdot \mathrm{b}\right]} \\
& {[\mathrm{A} \cdot \mathrm{a}] \stackrel{k_{\text {s.r. }}}{\longrightarrow}\left[\mathrm{A} \cdot \mathrm{a}^{*}\right]} \\
& \mathrm{BA}+\mathrm{a}^{*}+\mathrm{b} \underset{k_{\mathrm{off}}^{12 \mathrm{nt}}}{\stackrel{k_{\mathrm{on}}^{12 \mathrm{nt}}}{\rightleftharpoons}}\left[\mathrm{BA} \cdot \mathrm{a}^{*}\right]+\mathrm{b} \underset{k_{\mathrm{off}}^{12 \mathrm{nt}}}{\stackrel{k_{\mathrm{on}}^{12 \mathrm{nt}}}{\rightleftharpoons}}\left[\mathrm{BA} \cdot \mathrm{a}^{*} \cdot \mathrm{b}\right] \\
& \mathrm{BA}+\mathrm{a}^{*}+\mathrm{b} \underset{k_{\mathrm{off}}^{12 \mathrm{nt}}}{\stackrel{k_{\mathrm{on}}^{12 \mathrm{nt}}}{\rightleftharpoons}}[\mathrm{BA} \cdot \mathrm{b}]+\mathrm{a}^{*} \underset{k_{\mathrm{off}}^{12 \mathrm{nt}}}{\stackrel{k_{\mathrm{on}}^{12 \mathrm{nt}}}{\rightleftharpoons}}[\mathrm{BA} \cdot \mathrm{a} * \cdot \mathrm{b}] \\
& \mathrm{ab}+\mathrm{A}+\mathrm{B} * \underset{k_{\mathrm{off}}}{\stackrel{k_{\mathrm{on}}^{12 \mathrm{nt}}}{\rightleftharpoons}}[\mathrm{A} \cdot \mathrm{ab}]+\mathrm{B}^{*} \underset{k_{\mathrm{off}}^{12 \mathrm{nt}}}{\stackrel{k_{\mathrm{on}}^{12 \mathrm{nt}}}{\rightleftharpoons}}\left[\mathrm{B}^{*} \cdot \mathrm{A} \cdot \mathrm{ab}\right] \\
& \mathrm{ab}+\mathrm{A}+\mathrm{B}^{*} \underset{k_{\mathrm{off}}}{\stackrel{k_{\mathrm{on}}^{12 \mathrm{nt}}}{\rightleftharpoons}}\left[\mathrm{B}^{*} \cdot \mathrm{ab}\right]+\mathrm{A} \underset{k_{\mathrm{off}}^{12 \mathrm{nt}}}{\stackrel{k_{\mathrm{on}}^{12 \mathrm{nt}}}{\rightleftharpoons}}\left[\mathrm{B}^{*} \cdot \mathrm{A} \cdot \mathrm{ab}\right] \\
& \mathrm{A}+\mathrm{a} * \underset{k_{\mathrm{off}}^{12 \mathrm{nt}}}{\stackrel{k_{\mathrm{on}}^{12 \mathrm{nt}}}{\rightleftharpoons}}[\mathrm{A} \cdot \mathrm{a} *] \\
& \mathrm{B}^{*}+\mathrm{b} \underset{k_{\mathrm{off}}^{12 \mathrm{nt}}}{\stackrel{k_{\mathrm{on}}^{12 \mathrm{nt}}}{\rightleftharpoons}}\left[\mathrm{B}^{*} \cdot \mathrm{b}\right]
\end{aligned}
$$

However, B* and $\mathrm{a}^{*}$ cannot support ligation (eqn (18)-(21)), sequestering templates and replicates from the reaction.

To simulate the reaction curves, we solved 23 ODEs based on eqn (1)-(23) in MATLAB (Table S4 $\dagger$ ). We then performed a least squared curve fit of the ODE solution to our data with $k_{\text {s.r. }}$ as a fit parameter and the above approximated side reaction rate (Fig. 3c) as an initial value. For the upper and lower bounds of $k_{\text {off }}^{12 n t, 20^{\circ} \mathrm{C}}$ and $k_{\text {off }}^{24 n t, 20^{\circ} \mathrm{C}}$ value estimates (ESI $3 \dagger$ ), the fit produced the $k_{\text {s.r. }}$ values of $2.69 \mathrm{~h}^{-1}$ and $2.63 \mathrm{~h}^{-1}$, respectively. This was higher, but still in good agreement with the experimental data. The discrepancy between the fitted and originally approximated $k_{\text {s.r. }}$ values likely originated from the temperature dependence of the measured side reaction rate or from the effects of the EDC hydrolysis product, its urea, on the DNA duplex stability. Both effects were not explicitly modeled. The theoretical fit reproduced well the experimental behavior of the system (Fig. 2a, solid blue line).

When the data were fit in the absence of temperature cycling (Fig. 2b), the fitted side reaction rate was 10 times lower. This lower rate confirmed the expectation that the side reaction is strong at elevated temperatures even though the temperature spikes lasted seconds in the experiment.
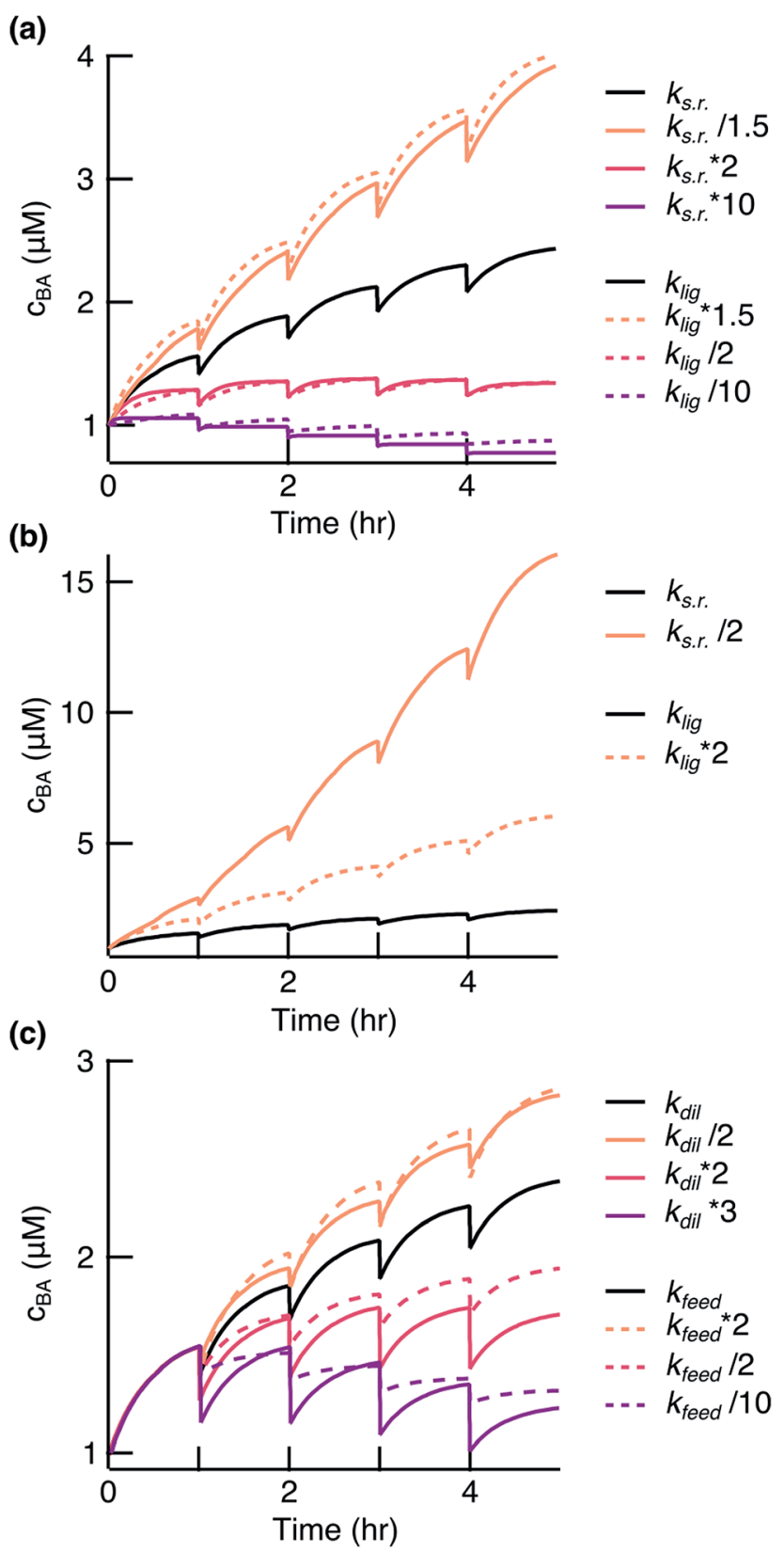

Fig. 5 Simulation of the ligation reaction. Black lines show the fit results to the experimental data. (a) The survival of the replicates depended on balancing $k_{\text {s.r. }}$ and $k_{\text {lig. }}$ (b) Decreasing $k_{\text {s.r. }}$ gave a stronger effect than enhancing $k_{\text {lig. }}$. (c) The survival of the replicates also depended on balancing feeding and dilution. 
The model is central to optimizing reaction conditions that support sustained replication. It shows that the replication system is very sensitive to the modulation of the ligation and side reaction rates. An increase in the side reaction rate and decrease in the ligation rate led to a system with a negative fitness (Fig. 5a, red and purple lines). However, an increase in the ligation rate or decrease in the side reaction rate by a factor of 1.5 significantly enhanced the replication (Fig. 5a, orange solid and dashed lines). The model also predicted that a decrease in the side reaction rate by a factor of 2 would result in a much stronger enhancement of replication compared to the effect from the increase in the ligation rate by the same factor (Fig. 5b). Since the side reaction rate showed such a strong inhibiting effect, the focus should be placed on finding ways to reduce it. This can be achieved by, for example, finding a condensing agent with less side product formation, e.g. diamidophosphate, ${ }^{31}$ a mechanism for a turnover of by-products, or a more gentle environment for strand separation.

Finding a balance between dilution and feeding is of equal importance. A higher dilution rate or less fragment feeding resulted in a negative fitness of the system (Fig. $5 \mathrm{c}$, red and purple lines). It could be of interest to decrease the dilution rate or increase the feeding to drive stronger amplification (Fig. 5c, orange lines). However, a too low dilution rate would lift the environmental pressure that we desire for fast evolution experiments. There is also a limit to how strong the feeding could be before feeding concentration of fragments becomes insoluble. We have also presented in the ESI $\dagger$ how other parameters affect the replication reaction (Fig. S6 $\dagger$ ). Taken together, the model will serve as a powerful tool to guide towards a faster and less wasteful replicator.

\section{Conclusions}

The presented DNA cross-replicator is a first but essential step towards open-ended evolution experiments from random oligonucleotides. In our system, 24mer strands replicated within a realistic timeframe and with linkage-chemistry enabling analysis by sequencing. Melting allowed the reuse of strands in the replication reaction leading to the survival of the system against the exponential dilution despite the side reactions. The presented reaction works on a defined set of DNA strands. However, it is expected that any two strands brought together by a template in the presence of EDC and $3^{\prime}$-phosphate groups would ligate. The efficiency of templated ligation between random strands would likely depend on the geometry and, thus, the sequence composition of the ligation site. ${ }^{32}$ The resulting longer molecules can then serve as templates in other reactions. The presented reaction opens a window to study how specific sequences were selected from random pools of short oligonucleotides likely present on the prebiotic Earth.

\section{Experimental}

\section{Materials}

$100 \mathrm{mM}$ MOPS at pH 6.5, adjusted with $\mathrm{NaOH}$, was used as the control buffer. $400 \mathrm{mM}$ EDC freshly dissolved in the control buffer was used as the reaction buffer. MOPS was purchased from Sigma Life Science (M1254-100G) and EDC-HCl was purchased from Carl Roth GmbH (Art.-Nr. 2156.2). Only nuclease-free water from Ambion (AM9932) was used in all experiments.

\section{DNA oligonucleotides}

All DNA oligonucleotides were purchased from biomers.net GmbH with HPLC purification. The sequences were as follows. Template BA: 5'-TTT ATT ATT TTA TAT TAT TTA TTT-3', template ab: $5^{\prime}$-AAA TAA ATA ATA TAA AAT AAT AAA- $3^{\prime}$, replicate $\sim$ BA: $5^{\prime}$-CCC TTT ATT ATT TTA TAT TAT TTA TTT- $3^{\prime}$, replicate ab: 5'-CCC CCC CCC AAA TAA ATA ATA TAA AAT AAT AAA-3', fragment $\sim$ B: $5^{\prime}$-CCC TTT ATT ATT TTA-3' (with or without a $3^{\prime}$ phosphate group for reaction or control experiments), fragment b: 5'-TAA AAT AAT AAA- $3^{\prime}$, fragment A: 5'-TAT TAT TTA TTT-3', fragment $\sim$ a: $5^{\prime}$-AAA TAA ATA ATA-3' (with or without a $3^{\prime}$ phosphate group for reaction or control experiments), normalization strand (Fig. S1a †): 5'-TAG TTA TGT CGA TAC GAG ACA ACA CCA TAT GCA TTT AAG TCG CTT GAA ATT GCT ATA AGC AGA GCA CTC GTA TCG ACA TAA CTA-3'.

\section{General reaction procedure}

The DNA was mixed on ice at a $1: 1$ ratio with $2 \times$ reaction buffer in a maximum recovery PCR tube (Axygen, a brand of Corning Life Sciences). Promptly, the tube was placed in a qTower ${ }^{3} \mathrm{G}$ cycler (Analytik Jena AG) at $20^{\circ} \mathrm{C}$ to start temperature cycling (with $8{ }^{\circ} \mathrm{C} \mathrm{s}^{-1}$ heating rate, $6{ }^{\circ} \mathrm{C} \mathrm{s}^{-1}$ cooling rate). For serial dilution, the cycler was paused 2 seconds before the end of the corresponding $20^{\circ} \mathrm{C}$ step and the tube was placed on ice. $10 \%$ of the reaction volume was precipitated for analysis and the volume was replaced with $5 \%$ volume of the fragment mix in water (each fragment at $20 \times$ its initial concentration) and with another $5 \%$ volume of the $2 \times$ reaction buffer. The tube was promptly placed back in the cycler, and the cycler program was continued. At the end of the reaction run time, the full sample volume was precipitated.

We performed the standard ethanol precipitation ${ }^{33}$ with addition of $2 \mu \mathrm{l}$ (for a sample volume of $30 \mu \mathrm{l}$ ) of glycogen $\left(10 \mathrm{mg} \mathrm{ml}^{-1}\right)$, which is known to enhance the precipitation of short oligonucleotides. We allowed the DNA to precipitate overnight at $4{ }^{\circ} \mathrm{C}$ before centrifugation, cleaning the pellet with $70 \%$ ethanol, and air drying it. The precipitation efficiency of more than $85 \%$ was quantified independently with standards.

\section{Reaction analysis method}

The precipitated samples were re-dissolved in $x / 50 \times v$ volume of water, where $x$ is the initial concentration of one of the templates and $v$ is the volume of the sample used for precipitation. The samples were loaded onto a Bioanalyzer chip (Agilent Small RNA Kit) and the Bioanalyzer assay was performed according to the company's protocol. Quantification was performed as explained in detail in ESI 1.† Quantification with the Agilent Bioanalyzer instrument established in this paper for ssDNA allowed us to quantify many samples in a parallel 
fashion using a minimal amount of material per analysis $(1 \mu \mathrm{l}$ of $50 \mathrm{nM}$ concentrated DNA strand per sample), roughly 100 times less than with HPLC.

All the concentration data from the Bioanalyzer instrument are presented with a $10 \%$ error bar, which was the pipetting error. We observed that after the data normalization and concentration calibration, the $10 \%$ pipetting error remained as the major error source (ESI 1) $\dagger$

\section{Melting curves}

Melting curves were measured using a C1000 ${ }^{\mathrm{TM}}$ Thermal Cycler with a CFX96 ${ }^{\mathrm{TM}}$ detection system (Bio-Rad Laboratories) at a rate of $0.083{ }^{\circ} \mathrm{C} \mathrm{s}^{-1}$ in either the control buffer or the reaction buffer. Eva Green dye (Biotium Inc.) was used at a $1 \times$ concentration for detection. Templates and replicates were measured at $1 \mu \mathrm{M}$ concentration each, while all fragments were measured at $10 \mu \mathrm{M}$ concentration each.

\section{Quantification of the dissociation rates}

Each melting curve was baseline corrected and normalized to depict the fraction of bound strands on the $y$ axis. The association constant, $K_{\mathrm{a}}$, was quantified from the Arrhenius plot following the description in the literature, ${ }^{34}$ using an in-house written LabVIEW routine (LabVIEW 2010, National Instruments). The dissociation rate, $k_{\text {off }}$, was then quantified from $K_{\mathrm{a}}$ $=k_{\text {on }} / k_{\text {off }}$, where the association rate, $k_{\text {on }}\left(0.4 \mu \mathrm{M}^{-1} \mathrm{~s}^{-1}\right)$, was taken from the literature..$^{26,27}$

\section{Simulation and data fitting}

The ODEs were solved in MATLAB using the solver ode15s. The experimental data were fitted with the lsqcurvefit tool with $k_{\text {s.r. }}$ as a fit parameter. The MATLAB code that allows running the simulation, fitting the data, and plotting the results is attached to this paper.

\section{Conflicts of interest}

There are no conflicts to declare.

\section{Acknowledgements}

Financial support came from the German Research Foundation (DFG) through Transregional Collaborative Research Center (SFB/Transregio) 235 Projects 07 and 16, the Simons Foundation (Grant Number 327125), and the Quantitative Biosciences Munich (QBM) Graduate School. We thank Georg Urtel and Eiman Osman for critical discussions related to this work, Stefanie Leiner for her expertise in chemistry, Matthias Morasch, Christina Dirscherl, Patrick Kudella, and Lorenz Keil for valuable comments on the manuscript.

\section{References}

1 W. Huang and J. P. Ferris, J. Am. Chem. Soc., 2006, 128, 89148919.
2 M. Morasch, C. B. Mast, J. K. Langer, P. Schilcher and D. Braun, ChemBioChem, 2014, 15, 879-883.

3 L. Da Silva, M. C. Maurel and D. Deamer, J. Mol. Evol., 2015, 80, 86-97.

4 P. A. Monnard, A. Kanavarioti and D. W. Deamer, J. Am. Chem. Soc., 2003, 125, 13734-13740.

5 M. Eigen and P. Schuster, Naturwissenschaften, 1977, 64, 541565.

6 W. Gilbert, Nature, 1986, 319, 618.

7 A. Wochner, J. Attwater, A. Coulson and P. Holliger, Science, 2011, 332, 209-212.

8 D. P. Horning and G. F. Joyce, Proc. Natl. Acad. Sci. U. S. A., 2016, 113, 9786-9791.

9 M. Sosson and C. Richert, Beilstein J. Org. Chem., 2018, 14, 603-617.

10 L. K. L. Cheng and P. J. Unrau, Cold Spring Harbor Perspect. Biol., 2010, 2, a002204.

11 J. Attwater, A. Raguram, A. S. Morgunov, E. Gianni and P. Holliger, eLife, 2018, 7, e35255.

12 H. Mutschler, A. Wochner and P. Holliger, Nat. Chem., 2015, 7, 502-508.

13 T. A. Lincoln and G. F. Joyce, Science, 2009, 323, 1229-1232. 14 G. von Kiedrowski, Angew. Chem., Int. Ed. Engl., 1986, 25, 932-935.

15 D. Sievers and G. Von Kiedrowski, Chem.-Eur. J., 1998, 4, 629-641.

16 J. W. Szostak, J. Syst. Chem., 2012, 3, 2.

17 G. von Kiedrowski, B. Wlotzka, J. Helbing, M. Matzen and S. Jordan, Angew. Chem., Int. Ed. Engl., 1991, 30, 423-426.

18 M. Jauker, H. Griesser and C. Richert, Angew. Chem., Int. Ed., 2015, 54, 14564-14569.

19 B. T. Burcar, M. Jawed, H. Shah and L. B. McGown, Origins Life Evol. Biospheres, 2015, 45, 31-40.

20 J. P. Ferris, G. Ertem and V. Agarwal, Origins Life Evol. Biospheres, 1989, 19, 165-178.

21 M. Todisco, T. P. Fraccia, G. P. Smith, A. Corno, L. Bethge, S. Klussmann, E. M. Paraboschi, R. Asselta, D. Colombo, G. Zanchetta, N. A. Clark and T. Bellini, ACS Nano, 2018, 12, 9750-9762.

22 T. N. Grossmann, A. Strohbach and O. Seitz, ChemBioChem, 2008, 9, 2185-2192.

23 C. B. Mast, S. Schink, U. Gerland and D. Braun, Proc. Natl. Acad. Sci. U. S. A., 2013, 110, 8030-8035.

24 M. Kreysing, L. Keil, S. Lanzmich and D. Braun, Nat. Chem., 2015, 7, 203-208.

25 R. Krishnamurthy, Acc. Chem. Res., 2017, 50, 455-459.

26 T. E. Ouldridge, P. Šulc, F. Romano, J. P. K. Doye and A. A. Louis, Nucleic Acids Res., 2013, 41, 8886-8895.

27 D. Y. Zhang and E. Winfree, J. Am. Chem. Soc., 2009, 131, 17303-17314.

28 A. Williams and I. T. Ibrahim, Chem. Rev., 1981, 81, 589-636.

29 N. Wrobel, M. Schinkinger and V. M. Mirsky, Anal. Biochem., 2002, 305, 135-138.

30 M. Tena-Solsona, B. Rieß, R. K. Grötsch, F. C. Löhrer, C. Wanzke, B. Käsdorf, A. R. Bausch, P. MüllerBuschbaum, O. Lieleg and J. Boekhoven, Nat. Commun., 2017, 8, 15895. 
31 C. Gibard, S. Bhowmik, M. Karki, E. K. Kim and R. Krishnamurthy, Nat. Chem., 2018, 10, 212-217.

32 K. Kawamura and F. Okamoto, Nucleic Acids Symp. Ser., 2000, 44, 217-218.
33 J. Sambrook, E. F. Fritsch and T. Maniatis, Molecular cloning: a laboratory manual, Cold Spring Harbor Laboratory, 1989.

34 J.-L. Mergny and L. Lacroix, Oligonucleotides, 2003, 13, 515537. 